\title{
fMRI activation during failures to respond key to understanding performance changes with sleep deprivation
}

\author{
Adrienne M. Tucker ${ }^{\mathrm{a}}$, Brian C. Rakitin ${ }^{\mathrm{a}, \mathrm{b}, \mathrm{c}}$, Robert C. Basner ${ }^{\mathrm{b}, \mathrm{e}}$, Yunglin Gazes ${ }^{\mathrm{a}}$, \\ Jason Steffener ${ }^{\mathrm{a}}$, Yaakov Stern ${ }^{\mathrm{a}, \mathrm{b}, \mathrm{c}, \mathrm{d}, \mathrm{f}, *}$ \\ a Cognitive Neuroscience Division of the Taub Institute for Research in Alzheimer's disease and the Aging Brain, 630 West 168th Street, PH-18, New York, NY 10032, United States \\ ${ }^{\mathrm{b}}$ Gertrude H. Sergievsky Center, New York, NY 10032, United States \\ ' Department of Neurology, College of Physicians and Surgeons of Columbia University, New York, NY 10032, United States \\ d Department of Psychiatry, College of Physicians and Surgeons of Columbia University, New York, NY 10032, United States \\ e Department of Medicine, College of Physicians and Surgeons of Columbia University, New York, NY 10032, United States \\ ${ }^{\mathrm{f}}$ Department of Psychology, College of Physicians and Surgeons of Columbia University, New York, NY 10032, United States
}

\section{A R T I C L E I N F O}

\section{Article history:}

Received 7 June 2010

Received in revised form 26 October 2010

Accepted 5 November 2010

\section{Keywords:}

Failures to respond

Individual differences

Lapses

Multivariate network analysis

Non-responses

Sleep deprivation

\begin{abstract}
A B S T R A C T
Study objectives: During sleep deprivation (SD), failures to respond (FR) increase across a variety of tasks. This is the first systematic investigation of neural correlates of FR during SD. We use multivariate analysis to model neural activation separately for FR and responses (R) at each trial phase.

Setting: In two experiments a delayed letter recognition task was performed in a 1.5T scanner at 9:30 am after two nights of total SD. Participants were continuously monitored in the laboratory.

Participants: Healthy young adults from two SD experiments (combined $n=37$; aged $25.55 \pm 3.86$ years). Materials and methods: Multivariate linear modeling (MLM) was used to find networks of activation that differed between FR and R. At each of three trial phases-encoding, retention, and test-two networks were expressed. In the encoding phase, the second network was seen during FR and was not seen during R. This network constituted widespread deactivations ( 26,000 voxels) of fronto-parietal and thalamic areas concomitant with activation of extrastriate cortex and hippocampus. In a multiple regression including activation during FR and $\mathrm{R}$ from all networks and all trial phases, expression of this encodingphase network during FR was the key predictor of SD-related performance impairment, operationalized as greater \%FR $\left(\eta_{\mathrm{p}}^{2}=0.33\right)$, lower $d^{\prime}$ and larger median RT $\left(\eta_{\mathrm{p}}^{2}=0.17\right)$.

Conclusions: FR were most associated with neural disruptions occurring at the encoding phase when subjects must attend to and encode items. Further, expression of this FR-related encoding-phase network made the largest independent contribution to predicting vulnerability to overall SD-related impairment.
\end{abstract}

(C) 2010 Elsevier B.V. All rights reserved.

\section{Introduction}

Failures to respond, or non-responses (sometimes called errors of omission), have been noted to increase during sleep deprivation on a variety of tasks: serial subtraction [1], psychomotor vigilance [2], arrow flanker [3], semantic judgment [4], as well as working memory tasks [5-8]. Understanding such failures to respond would seem to provide much insight into the disproportionate number of

\footnotetext{
* Corresponding author at: Columbia University, 630 West 168 th Street, P \& S Box 16, New York, NY 10032, United States. Tel.: +1 212342 1350; fax: +1 2123421838.

E-mail addresses: at2573@columbia.edu (A.M. Tucker), br130@columbia.edu (B.C. Rakitin), rcb42@columbia.edu (R.C. Basner), yl2107@columbia.edu (Y. Gazes), js2746@columbia.edu (J. Steffener),ys11@columbia.edu (Y.Stern).

URL: http://www.cumc.columbia.edu/dept/sergievsky/cnd/stern.html (Y. Stern).
}

real-world accidents that have been attributed in part or in whole to sleep deprivation [9]. This is the first report focused on the neural correlates of failures to respond during sleep deprivation.

Two previous articles reported on failures to respond during sleep deprivation, although this was not the main focus of either article $[4,5]$. Non-responses seemed to be associated with less activation of task-relevant areas as well as less activation of some additional areas. In these studies, however, activation was pooled across responded to and non-responded to trials, which may have obscured any neural activation that is only present during failures to respond.

We report here the first systematic investigation of neural correlates of failures to respond during sleep deprivation. Specifically, we examine networks of expression that differ between responded to and non-responded to trials on a delayed letter recognition task. The event-related design allows us to separate the encoding, retention, and test phases of each trial. We further investigate how 
expression of responded to and of non-responded to trials at each of these trial phases correlates with the performance decrement with sleep deprivation in terms of speed, accuracy, and non-responses.

\section{Materials and methods}

\subsection{Participants}

Participants were from two sleep deprivation (SD) experiments ( $n=17$ and $n=20$ ). While changes were made to other experimental tasks, the delayed letter recognition task (DLR) was run according to the same protocol in each study. Younger adults were recruited from the community using flyers; age range was 20-35 (25.55 $\pm 3.86 ; 30$ male, 7 female). All participants were right-handed with normal or corrected-to-normal vision and screened for medical and psychiatric disorders, the presence of a sleep disorder, and/or any substance abuse. Partial data from subjects from the first and/or second experiment were used in the cited reports, none of which focused on the present topic [5,10-13]. The experiment was performed with the understanding and written consent of each subject.

\subsection{Task}

The critical experimental factor for this DLR task was set size, which is the number of letters (either 1,3 , or 6 ) to be remembered on each trial. Set size was varied pseudo-randomly across trials. Each of three experimental blocks contained 10 trials at each of the three set sizes, with five true negative (i.e., non-matching) probes and five true positive (i.e., matching) probes per set size. In sum, there were a total of 30 trials at each of the three blocks for a total of 90 experimental trials per subject.

The sequence of trial events was as follows: first, a fixed blank inter-trial interval (ITI) of $3 \mathrm{~s}$; then, a memory set of 1,3 , or 6 letters was presented for $3 \mathrm{~s}$; next, there was a delay of $7 \mathrm{~s}$ during which the memory set had to be retained; finally, the probe was on the screen until the participants responded or $3 \mathrm{~s}$ had passed, whichever came first. In addition to the $3 \mathrm{~s}$ ITI, there were also 70 two-second intervals per block that were inserted in a random fashion between trials. For more details see [5].

\subsection{Protocol}

Participants were continuously monitored in the laboratory. They were required to abstain from caffeine for $24 \mathrm{~h}$ prior to study participation and for the duration of the study. Participants kept sleep logs for two weeks prior to laboratory entry. Participants in the first SD study slept $7.8 \pm 1 \mathrm{~h}$ per night; in the second SD study one subject had missing data, the other participants slept $8.1 \pm 0.7 \mathrm{~h}$ per night. For both studies, the protocol for the DLR was the same. All participants received one training session with feedback prior to the initial scanned session. At this session they received 7 blocks of 30 trials; for the first 6 blocks feedback was provided while for the final block no feedback was provided. Then, the initial scanning session occurred at $9 \mathrm{am}$ and the follow-up scanning session occurred at the same time $48 \mathrm{~h}$ later, to control for known circadian influences on the effects of sleep deprivation [14]. For more details of the protocol see [15].

\section{4. fMRI acquisition and preprocessing}

During the performance of each block of the DLR, 207 blood-oxygen-leveldependent (BOLD) images $[16,17]$ were acquired with an Intera 1.5T Phillips MR scanner equipped with a standard quadrature head coil, using a gradient echo echoplanar (GE-EPI) pulse sequence $(\mathrm{TE} / \mathrm{TR}=50 \mathrm{~ms} / 3000 \mathrm{~ms}$; flip angle $=90 ; 64 \times 64$ matrix, in-plane voxel size $=3.124 \mathrm{~mm} \times 3.124 \mathrm{~mm}$; slice thickness $=8 \mathrm{~mm}$ (no gap); 17 trans-axial slices per volume). Four additional GE-EPI excitations were performed before the task began, at the beginning of each run, to allow transverse magnetization immediately after radio frequency excitation to approach its steady-state value; the images corresponding to these excitations were discarded. Data were spatially normalized using a T1-weighted spoiled gradient image (107 slices; $256 \times 256$ grid; FOV $=230 \mathrm{~mm} \times 160.5 \mathrm{~mm} \times 183.28 \mathrm{~mm}$ )

Task stimuli were back-projected onto a screen located at the foot of the MRI bed using an LCD projector which participants viewed via a mirror system located in the head coil. All participants wore MR compatible glasses as needed to have vision at their best corrected acuity (manufactured by SafeVision, LLC. Webster Groves, MO). Responses were made on a LUMItouch response system (Photon Control Company) using the index fingers of either hand. Task administration and collection of response data were controlled using PsyScope 1.2.5 [18] running on a Macintosh G3 iBook. Task onset was electronically synchronized with the MRI acquisition computer. A Carnegie Mellon Button Box (New Micros, Inc. Dallas, TX) provided digital input-output for the response system and synchronization with the MRI acquisition computer, as well as millisecond accurate timing of responses.

\section{5. fMRI time-series (i.e., first-level) modeling}

Time series modeling had regressors representing activity of the three trial phases-encoding, maintenance and test-separately for each set size $(1,3$, or 6 letters). One rectangular regressor was used for each of the trial components: encoding
( $3 \mathrm{~s}$ in duration), maintenance (7s in duration), and test ( $3 \mathrm{~s}$ in duration). Failures to respond (FR), which refer to trials without motor responses from the participant during the $3 \mathrm{~s}$ test period, were modeled separately. Additionally, trials where an incorrect response was made were modeled separately.

\subsection{Multivariate linear modeling (MLM)}

The goal of our analysis was to contrast brain activation during $\mathrm{R}$ with that observed during FR. As FR were negligible during baseline, this analysis was restricted to the fMRI data collected during sleep deprivation. The spatial networks that differed between $\mathrm{R}$ and FR during sleep deprivation were assessed via the application of the multivariate linear modeling (MLM) theory [19]. Specifically, MLM was used to determine if the group-mean contrast images (here the contrast was the slope across set size, which presumably reflects task-specific processes) could be expressed as linear combinations of one or more latent spatial variables, or networks. Singular-value decomposition (SVD) was performed for the encoding, retention, and test phases separately. Each SVD examined 1 effect of interest, which was the group mean contrast images for R and FR trials, respectively, generated by random effect group level General linear modeling (GLM) [20] within SPM5.

Sequential latent root testing, using a global $F$-test and an alpha level of 0.05 , is used to determine the number of significant spatial networks. The maximal number of spatial networks is determined by the dimensionality of the F-contrast. Each $F$ contrast compares $\mathrm{R}$ and FR trials and therefore has a dimensionality of two. Thus, the largest number of potentially significant spatial networks is two for each of the encoding, retention, and test phases.

The areas comprising each MLM network were represented visually, scaled by their singular values, as $\operatorname{SPM}(t)$. $\operatorname{SPM}(t)$ maps are presented with thresholds of a $t$-value corresponding to $p<0.001$ uncorrected for multiple comparisons, and a minimum cluster $(k)$ size of 50 voxels. These values are chosen simply to select the most prominent areas in the spatial networks. The Talairach coordinates and their anatomical labeling based on the template created with automatic anatomical labeling [21] were reported for local maxima of $Z$-scores in each network.

We calculated the observed expressions of each network by participants for $\mathrm{R}$ and FR trials, respectively. We summarized these observed expressions with a single value per participant (the individual subject expression) by taking the inner product of a vector of the observed expression values for that subject and a vector of the predicted expression values over conditions for that subject. In sum, each subject had a single value for network expression during $\mathrm{R}$ trials and likewise a single value for network expression during FR trials for each trial phase-encoding, retention, and test. These summarized observed expressions were used as dependent variables in a multiple regression to test hypotheses regarding the association between activation of the networks and behavioral performance impairments with SD (see below).

\subsection{Brain-behavior analyses}

Two multivariate general linear models were used-the full and the reduced model. For all models our dependent variables (DVs) constituted the change in performance from the first to the second session (i.e., the change in performance with SD) for (1) percent FR, (2) $d^{\prime}$, a measure of accuracy and (3) median RT. For the full model, the independent variables (IVs) were Group (i.e., the first SD group versus the second SD group), and expression of the spatial networks found in the MLM analysis described above: that is, the expression of each spatial network found in each condition ( $R$ versus FR trials) at each phase (encoding, retention, and test). As we found two networks for each of two trial types at three test phases this yielded 12 network expression scores (Encoding Network 1 during R, Encoding Network 1 during FR, etc.). We also initially included the crossing of group with each of the 12 network expression scores as IVs. As none of these crossings were significant, our reduced model dropped these 12 crossings and only retained the main effect of Group. Significance was assessed with the approximate $F$ derived from Wilke's $\Lambda$, with $\alpha=0.05$. If the multivariate $F$ was significant, than any significant univariate $F s$ were interpreted.

\section{Results}

\subsection{Group behavioral results}

As expected, during SD participants were slower, less accurate, and displayed more FR on the DLR. Specifically, participants slowed down an average of $158 \pm 32 \mathrm{~ms}$ during SD; further, $d^{\prime}$ values fell by $1.3 \pm 0.2$, and the percentage of FR increased significantly from $1 \pm 0.4 \%$ to $31 \pm 16 \%$. The increase in FR was not correlated with the increase in median RT $(r=0.005, p=0.98)$, indicating that the greater number of FR during SD were not just an artifact of slowing. The increase in FR was, however, correlated with the decrease in accuracy $(r=-0.46, p=0.0002)$. 
A Stimulus Phase Network Expression

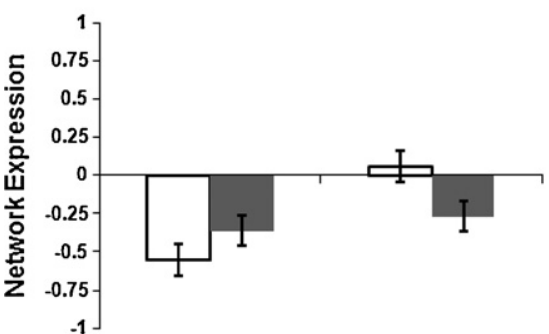

Stm Network1 Stm Network 2

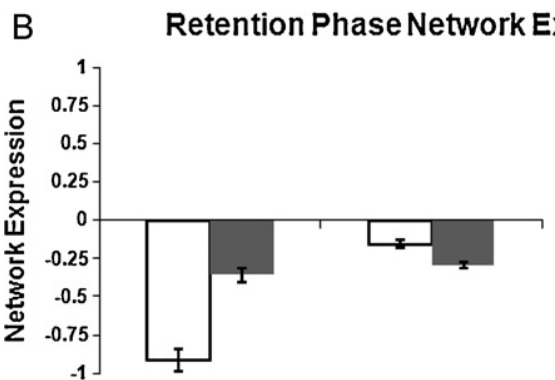

Ret Network 1 Ret Network 2

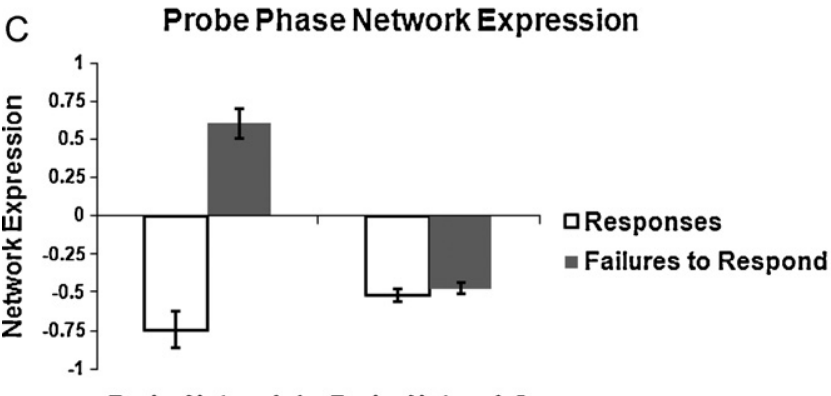

Probe Network 1 Probe Network 2

Fig. 1. fMRI network expression during responses and failures to respond. Mean network expression across subjects and standard error are displayed for each network in (A) the encoding phase; (B) the retention phase; and (C) the test phase. The $Y$-axis scale is in arbitrary units.

\subsection{MLM analysis: neural network results}

Encoding phase: During the encoding phase, the MLM analysis comparing activation in $\mathrm{R}$ and FR identified 2 significant spatial networks. We calculated network expression in each subject for both $\mathrm{R}$ and FR. These are illustrated in Fig. 1. The areas comprising each MLM network are represented visually in Fig. 2. Additionally, precise Talairach coordinates for local maxima of $Z$-scores are reported as supplementary information (Supplementary Tables S1 and S2).

Network 1 was expressed in both in R and FR. However, it was expressed significantly more in $\mathrm{R}$ than in FR (K-S, $p<0.0001)$. Thus, Network 1 revealed that for the encoding phase, $R$ and, to a lesser extent, FR, were associated with a network of brain regions that included 1) activations in the thalamus and lingual gyrus and 2) deactivations in the extrastriate cortex, anterior cingulate, and middle and inferior frontal gyri. See Figs. 1 and 2 and Supplementary Table S1.

Network 2 was expressed significantly differently between $\mathrm{R}$ and FR (K-S, $p=0.005)$. The average network expression for $\mathrm{R}$ was near zero, suggesting that this network was expressed during FR only. Specifically, FR were associated with a network that involved (1) activations in extrastriate cortex and hippocampus and (2) deactivations across a broad range of areas including the thalamus,
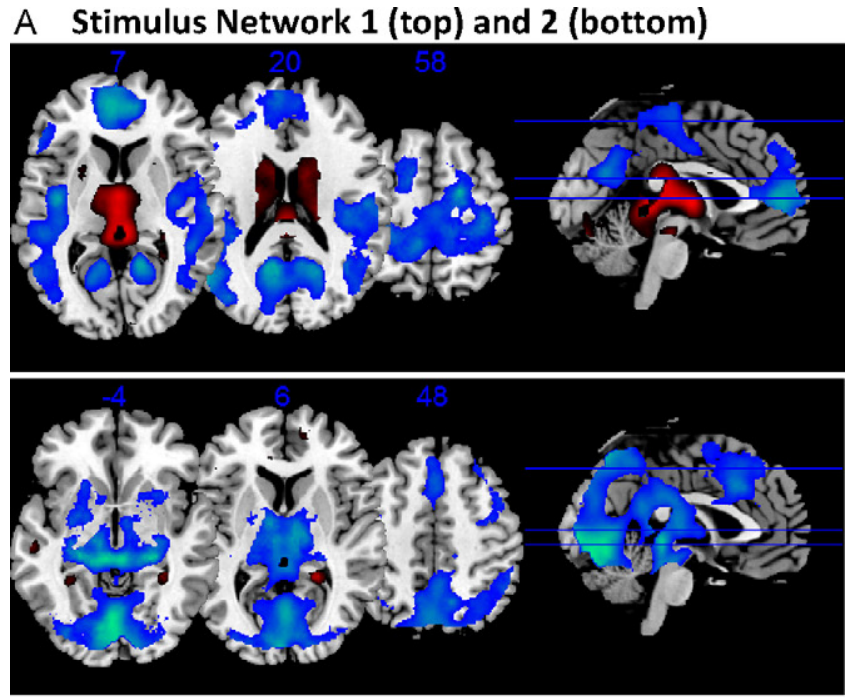

B Retention Network 1 (top) and 2 (bottom)
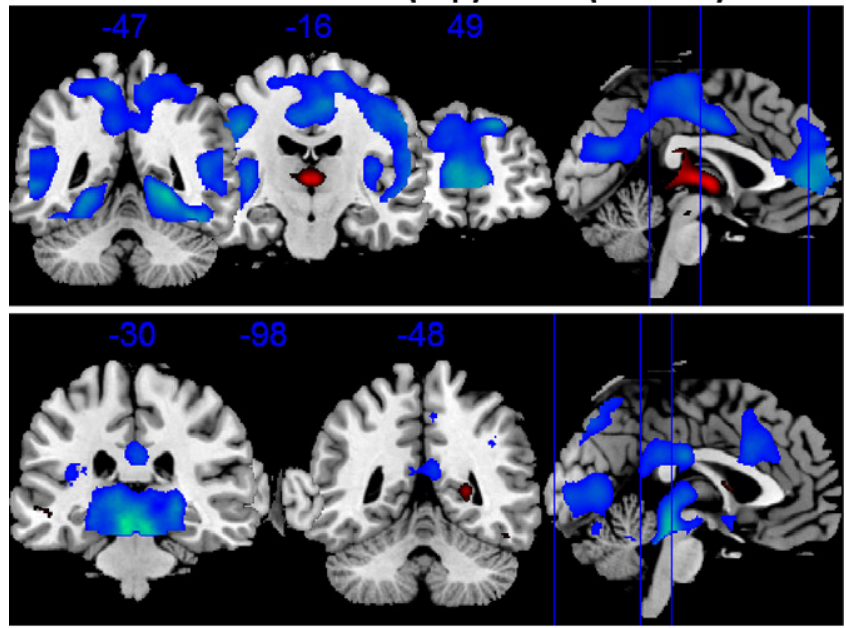

C Probe Network 1 (top) and 2 (bottom)
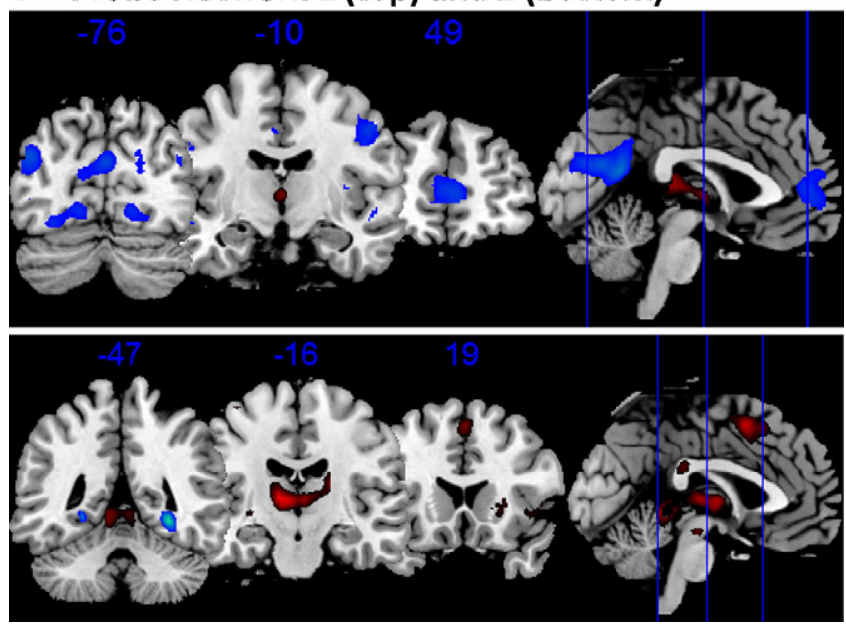

Fig. 2. Regions of network activation. $\operatorname{SPM}(t)$ maps are presented with thresholds of a $t$-value corresponding to $p<0.001$ uncorrected for multiple comparisons, and a minimum cluster $(k)$ size of 50 voxels; for the first network of the test phase activation was the highest and this first test network was therefore thresholded at $p<0.0001$. Red indicates areas more activated than during rest; blue indicates areas less activated than during rest. 


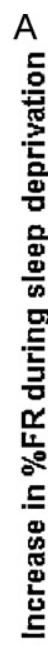

$-1.1$

$-0.6$

$-0.1$

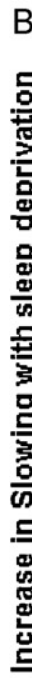

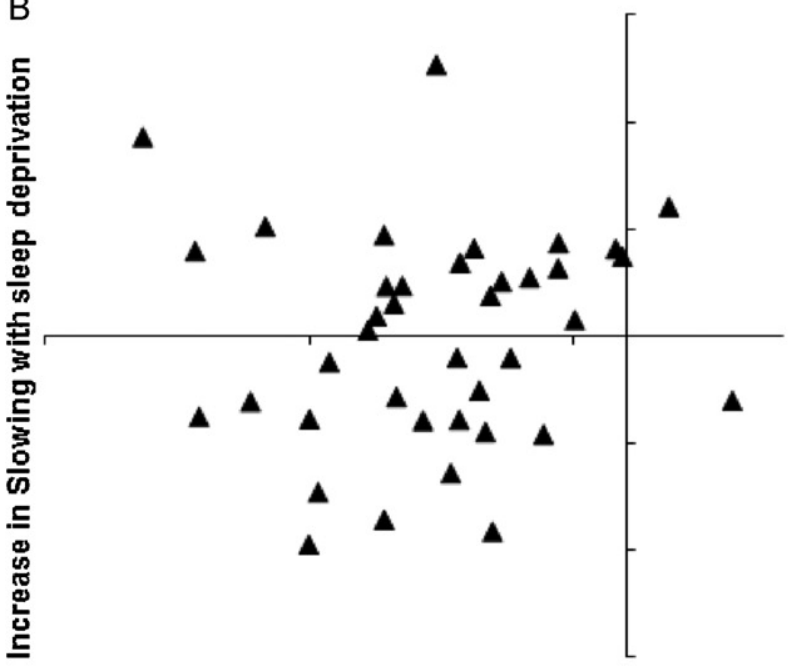

$-1.1-0.6$

$-0.1$

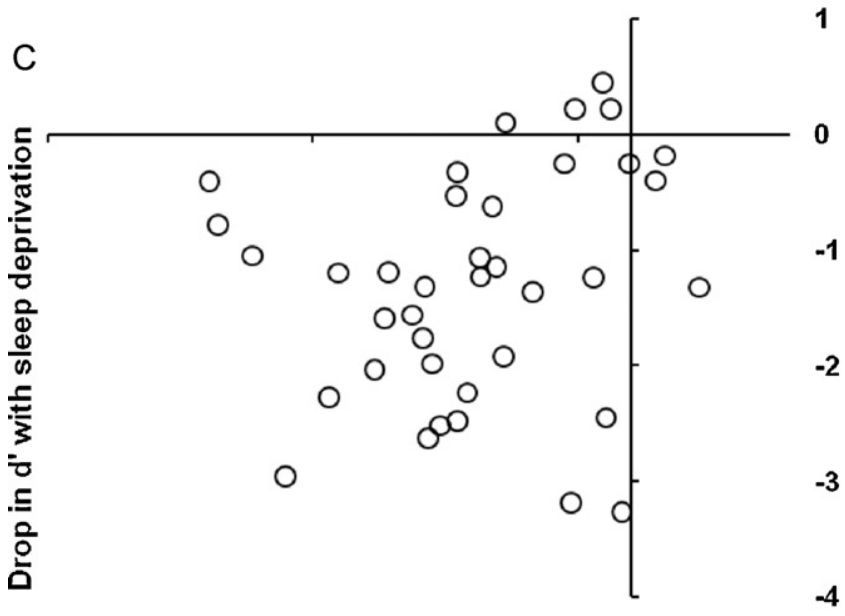

$\begin{array}{lll}-1.1 & -0.6 & -0.1\end{array}$

Expression of Stimulus Network 2 during FR lingual gyrus, cingulate gyrus, medial and superior frontal gyri, precentral gyrus, superior and inferior parietal lobule, angular gyrus, and the precuneus. See Figs. 1 and 2 and Supplementary Table S2.

Retention phase: Two significant networks were identified in the retention phase (Figs. 1 and 2 and Supplementary Tables S3 and S4). Network 1 was expressed during both $\mathrm{R}$ and FR. This network was expressed relatively more for $\mathrm{R}$, however, and this difference was significant $(p<0.0001)$. The regions that made up this network included: (1) activations in the thalamus, the caudate body, and the caudate tail and (2) deactivations in the middle temporal gyrus, the parahippocampal gyrus, and the lingual gyrus.

Network 2 was also expressed for both R and FR. There was a trend for the network to be more highly expressed for $\operatorname{FR}(p=0.066)$. This network involved (1) activations in parahippocampal gyrus, inferior and superior frontal gyri, and the caudate body and head and (2) deactivations in the thalamus, red nucleus of the brainstem, transverse temporal gyrus, superior parietal lobule, supramarginal gyrus, precuneus, cuneus, anterior and posterior cingulate, insula, and the inferior and middle frontal gyri. See Figs. 1 and 2 and Supplementary Table S4.

Test phase: Two significant networks were identified in the test phase (Figs. 1 and 2 and Supplementary Tables S5-S6). Expression of Network 1 was significantly different for R and FR (K-S, $p<0.0001$ ), which were on opposite sides of zero (so in the opposite direction of one another). $\mathrm{R}$ were associated with (1) activations in the pulvinar of the thalamus and the caudate tail and (2) deactivations in the mammillary body, precuneus, and the posterior cingulate. For FR this pattern is reversed (i.e., deactivations in the pulvinar and caudate tail and activations in the mammillary body, precuneus, and the posterior cingulate).

Expression of Network 2 was not significantly different between $\mathrm{R}$ and $\mathrm{FR}$ (K-S, $p=0.92$ ). $\mathrm{R}$ and FR were both associated with (1) activations in the thalamus, parahippocampal gyrus, precentral gyrus, inferior and medial frontal gyrus, cingulate gyrus, caudate body, the lentiform nucleus of the putamen, and the superior parietal lobule and (2) deactivations in the parahippocampal gyrus. See Figs. 1 and 2 and Supplementary Table S6.

\subsection{Brain-behavioral results}

A multiple regression was performed to look for any associations between the change in behavioral performance during SD and expression of any of the neural spatial networks. The behavioral variables were \% FR, median RT, and $d^{\prime}$. Neural activation was available for R and FR trials separately at encoding, retention, and test phases. As each of these phases had two networks this yielded 12 neural spatial networks.

Our full model confirmed the absence of any interactions between SD group and expression of any of the 12 neural spatial networks. Our reduced model had an R-square value of 0.57 with $p=0.022$. See Supplementary Table S7 for a summary of $F$ values for all effects. Significant multivariate effects were as follows: group predicted median RT such that change in median RT with SD was higher in the second SD group (Partial Eta Squared $=0.27$ ); greater activation of the second network of the encoding phase in $\mathrm{R}$ (i.e., Stm_Eig2_R) correlated with a bigger drop in accuracy with SD as measured by change in $d^{\prime}$ (Partial Eta Squared $=0.23$ ); greater activation of Stm_Eig2_FR correlated with bigger decrements during SD for all three behavioral variables (Partial Eta Squared $=0.33$

Fig. 3. Brain-behavior relationships. Observed expressions of encoding network 2 during failures to respond are plotted on the $x$-axis of each graph. The $y$-axis of each graph displays the $Z$-scores of (A) the increase in \%FR with sleep deprivation; (B) the increase in slowing with sleep deprivation; and $(C)$ the drop in accuracy with sleep deprivation. 
for percent FR, 0.25 for $d^{\prime}$, and 0.17 for median RT; see Fig. 2); and, greater activation of Ret_Eig2_FR correlated with a bigger increase in FR with SD (Partial Eta Squared =0.24). See Fig. 3.

\section{Discussion}

This is the first systematic investigation of neural correlates of failures to respond during sleep deprivation. Healthy young adults were scanned while performing a working memory task of delayed letter recognition before and after $48 \mathrm{~h}$ of sleep deprivation. The expected performance impairment in terms of reduced speed and accuracy and increased percentage of FR was observed during sleep deprivation. Here we used a multivariate analysis to compare the recruitment of neural networks between FR and R during sleep deprivation. This was done separately for the encoding, retention, and test phases of each trial. We found that FR during sleep deprivation originated during the earliest, encoding phase of the trial. Further, neural activity during FR at this phase was the largest independent predictor of vulnerability to overall performance impairments during sleep deprivation. As such, expression of this network may constitute a biomarker for vulnerability to sleep loss.

\subsection{Neural networks and behavior}

At each of the three trial phases we found two networks expressedin a total of six networks. As expected from previous literature of working memory tasks [4,5], fronto-parietal, visual, and thalamic regions were heavily implicated. For detailed information about the regions involved in each network please consult the figures and supplementary tables.

A multiple regression was performed to examine any associations between change in behavioral performance during sleep deprivation and expression of any of the spatial networks. For each subject and each of the six networks (two at each of three task phases), there was one score representing the expression of a given network averaged across FR trials and a second score representing the expression of a given network averaged across $\mathrm{R}$ trials. This analysis thus contained 12 neural predictors and 3 behavioral outcome variables (\%FR, $d^{\prime}$, and median RT).

Greater expression of the second encoding phase network during FR was independently associated with greater SD-related impairment in all 3 behavioral variables. This suggests that activation of this network may constitute a biomarker for overall vulnerability to sleep loss. This network involved lesser activation of fronto-parietal, thalamic, and visual areas-superior and medial frontal gyrus, superior and inferior parietal lobule, thalamus, and the lingual gyrus-alongside greater activation of extrastriate cortex and hippocampus. Activation of the second retention phase network during FR was also independently associated with greater \%FR, albeit to a lesser extent. This network involved activations of dorso- and ventrolateral prefrontal gyrus, middle temporal and parahippocampal gyrus, and the caudate body and head; concomitant with deactivations in the superior frontal gyrus, anterior and posterior cingulate, precuneus and cuneus, insula, and thalamus.

While speculative, it could be that activation during the encoding phase is most strongly associated with performance because if an item is not successfully encoded, then the subject will necessarily not be able to successfully retrieve the item. This also supports Chee's hypothesis that deficits in visual processing underly working memory deficits during sleep deprivation. Activation during the retention period accounted for some unique variance, albeit to a lesser extent. This may indicate that some items were successfully encoded but failed to be maintained in working memory throughout the retention period. Activation during the retrieval period did not correlate with FR. This is in line with our previous behavioral results which indicated that the components of cognitive functioning operating during this phase-i.e., scanning through working memory, deciding yes or no, and making the motor response-are not affected by sleep deprivation $[5,7,22]$.

\subsection{Extending previous literature}

While not the primary focus, two previous articles report some correlates of FR during sleep deprivation [4,5]. As part of a larger investigation, Chuah et al. [4] reported on the magnitude of activation in task-relevant regions during a semantic judgment task (living or non-living). 26 healthy young participants (13 males) were scanned four times: two replications of a scan at 6:30 am of a single night of sleep deprivation, and two replications of a scan at 9:00 am after a night of sleep. Participants took either donepezil or placebo daily for at least 7 days prior to each scan. Whether sleep deprivation was prior to or after the rested session was counterbalanced within participants.

Fronto-parietal and visual areas emerged as important for this task: specifically the inferior frontal gyrus, inferior and superior parietal cortex, and the fusiform gyrus. Less activation in taskrelated areas during SD was associated with more FR. There were some fronto- parietal areas: the middle frontal gyrus and intraparietal sulcus-that were also implicated indirectly in FR as they were associated with the effect of donepezil administration in reducing FR. Of the above regions, our FR-related encoding-phase network included the inferior and superior parietal areas.

Habeck et al. (2004) scanned 18 healthy young participants after a night of sleep at 9:00 am and again at 9:00 am two days later after $48 \mathrm{~h}$ of sleep deprivation during performance of a working memory task of delayed letter recognition (DLR). This constituted the first of the two studies whose data are analyzed in the present report. Using a different multivariate technique than that used here, ordinal trends analysis, a network of brain regions was found that changed expression with sleep deprivation in as many participants as possible. This network was comprised of deactivation of parietal and visual processing regions-superior and inferior parietal areas and ventral stream visual areas-alongside activation in the anterior cingulate gyrus and thalamus. Although not the focus of the article, it was noted that the increase in network expression pre to post could predict the increase in the FR pre to post as well as other impairments including the drop in accuracy and the increase in response time variability. The activation here in superior and inferior parietal regions was also seen in the above Chuah et al. study and in the present analyses as well.

Taken together, the two above studies suggest that FR are associated with less activation of task-relevant areas, particularly parietal and visual areas. Regions associated with FR across the above two studies and the present analyses are the inferior and superior parietal areas. For the first time we model activation during FR separately rather than pooling across FR and $\mathrm{R}$ trials. We find that during the encoding phase FR are associated with less activation of fronto-parietal, thalamic, and visual areas-superior and medial frontal gyrus, superior and inferior parietal lobule, thalamus, and the lingual gyrus-but also with activation of extrastriate cortex and hippocampus.

\subsection{FR in relation to slowest responses}

Both FR and abnormally slow responses are grouped together as lapses by sleep researchers [22]. Indeed, the definition of a lapse as "the failure to respond in a timely fashion to a presented stimulus" covers both abnormally slow responses as well as non-responses. It has been observed that the longer a lapse is, the more likely it is to involve slow eyelid closures and/or entry into microsleep (i.e., 
brief periods of sleep that intrude into wakefulness under periods of sleep deprivation) [22]. Thus, the handful of studies involving neural modeling of lapses during sleep deprivation discarded FR in order to avoid including trials that involved eyelid closures and microsleep [23-25].

Here we do not discard FR but instead model them directly as, from our perspective, slow eyelid closures and microsleep do not reflect random nuisance but instead reflect the experimentally manipulated effect of interest, increasing sleep pressure. As such, neural activity during these trials may-in part-reflect sleepinitiating processes known to contribute to cognitive impairments during sleep deprivation [9]. We note that the areas we identify as related to FR do not overlap with those reported to occur during onset to stage one sleep in one previous study using rested subjects [25] yet it is possible that sleep onset looks differently during sleep deprivation. Even if, however, as is likely, subjects were experiencing such eyelid closures and microsleeps on some trials we do not see this as a problem but instead as evidence of the success of our experimental manipulation.

In this section, we compare our results to those obtained in the handful of investigations that have been conducted on the neural signature of the slowest responses during sleep deprivation. Specifically, these studies examined neural activation during the $10 \%$ slowest responses and during the $10 \%$ fastest responses as compared to the middle $80 \%$ of responses during sleep deprivation $[23,25]$. Note that for these studies FR were discarded. Chee et al. [23] scanned 24 young individuals at 6:00 am after a night of SD and one week later at 8:00 am when rested; the order of these two sessions (i.e., SD versus wake) was counterbalanced between subjects. The authors found that the slowest responses during sleep deprivation on a global-local letter identification task were associated with decreased peristimulus activation (1.5-3 s after stimulus onset) in fronto-parietal areas-the medial frontal cortex and intraparietal sulcus-alongside an increased peak signal in these areas later on in the trial. Further, the thalamus and extrastriate cortex showed decreased activation throughout the trial during the slowest responses. Chee et al. interpreted their findings in line with wake-state instability theory. That is, they explained the slowest RTs as arising with transient depressed activation that may be caused as an interaction between sleep-promoting mechanisms and voluntary mechanisms engaged to stay awake which interferes with attentional mechanisms. Specifically, they implicated a failure of selective attention to augment visual sensory processing, particularly extrastriate visual areas.

Drummond et al. [25] scanned 20 young subjects between 6:00 pm and 8:00 pm after a night of rest and at a second session between $6: 00 \mathrm{pm}$ and 8:00 pm after $36 \mathrm{~h}$ of SD; the order of SD and wake was counterbalanced between subjects. The authors found differences in activation between the slowest and the fastest $10 \%$ of responses utilizing the Psychomotor Vigilance Task, which measures simple RT. Drummond et al. found a greater magnitude of activation in fronto-parietal, thalamic, and visual processing areas (here the medial and middle frontal gyrus, superior and inferior parietal lobe, and the cuneus). This is parallel to what Chee et al. found except that in the Chee et al. study there was lesser activation in thalamic and visual processing areas. Again, the magnitude of activation was associated with the degree of vulnerability in performance. Two interpretations were put forth to explain these greater activations with lapsing. The first was that these activations of regions involved in the default network indicate that sleep deprived participants may sometimes fail to disengage processing away from the default network; this failure to overcome default network processing was theorized to produce slow responses. The second explanation was that activation of these areas represented a compensatory response immediately following the detection of the slow response. Like Chee et al., Drummond et al. also hypothe- sized that the differences in activation between the slowest and the fastest responses are a manifestation of state-instability theory.

Chee and Tan [24] investigated slowest responses during sleep deprivation using a global-local letter identification task similar to the one they reported on in their earlier study outlined above; they further performed a median split to divide subjects into those vulnerable and those non-vulnerable to sleep deprivation on the basis of performance accuracy. As before, they found that lapses were associated with an increase in parietal activation-intraparietal sulcus-and a decrease in thalamic activation. The extrastriate cortex showed the biggest drop in activation with sleep deprivation across all trials; this lowered activation did not differ between lapses and non-lapses. Vulnerable people did not display increased activation in their intraparietal sulcus during lapses, and showed more of a decrease in thalamic activation during lapses. Also, vulnerable participants had lower extrastriate activation overall. The authors interpreted the results as reflecting a failure during sleep deprivation in vulnerable individuals of top-down selective attentional control of sensory processing.

In accord with previous studies, we find that lapses are associated with changes in fronto-parietal, thalamic, and visual processing regions and that these changes are highest in the most vulnerable individuals. Thus, in spite of previous concerns about the suitability of including FR, the results here when examining FR directly are similar to those found when discarding FR. The current study confirms that these changes in activation are occurring during the earliest phases of each trial (i.e., the encoding phase). This finding is in line with previous reports that the effects of sleep deprivation on working memory tasks have more to do with attention and encoding than on memory storage and/or retrieval $[5,7]$.

\section{Summary}

This constitutes the first analysis focusing on the neural signatures of FR, known to increase during sleep deprivation across a variety of tasks. The great degree of overlap of brain activation between $F R$ and $R$ trials suggests that task-relevant processing was going on during FR trials. Additionally, FR were uniquely associated with a network comprised of widespread deactivations including fronto-parietal and thalamic areas concomitant with activation of extrastriate cortex and hippocampus; this network was expressed during the encoding phase of the trial. Moreover, expression of this network during FR made the largest independent contribution to predicting performance impairments during sleep deprivation for all variables examined: FR rate, accuracy, and median RT. Thus, in this first investigation of FR activation we have demonstrated that these trials contain important and interesting new information about the effects of sleep deprivation on cognition. Indeed, studying FR activation may provide traction in understanding and preventing safety-critical performance lapses. Additionally, FR activation may constitute a biomarker for individual vulnerability to these adverse events during conditions of sleep loss.

\section{Conflict of interest}

None of the authors have any conflicts of interest.

\section{Acknowledgements}

This work was supported by the Defense Advanced Research Projects Agency (DAAD 19-02-01-01147) and the National Institutes of Aging at the National Institutes of Health (T32 AG00261 and R01 AG026158). 


\section{Appendix A. Supplementary data}

Supplementary data associated with this article can be found, in the online version, at doi:10.1016/j.bbr.2010.11.012.

\section{References}

[1] Drummond SPA, Brown GG, Stricker JL, Buxton RB, Wong EC, Gillin JC. Sleep deprivation-induced reduction in cortical functional response to serial subtraction. Neuroreport 1999;10:3745-8.

[2] Doran SM, Van Dongen HPA, Dinges DF. Sustained attention performance during sleep deprivation: Evidence of state instability. Arch Ital Biol 2001:139:253-67.

[3] Hsieh S, Tsai C-Y, Tsai L-L. Error correction maintains post-error adjustments after one night of total sleep deprivation. J Sleep Res 2009;18:159-66.

[4] Chuah LYM, Chong DL, Chen AK, Rekshan WR, Tan J-C, Zheng H, et al. Donepezil improves episodic memory in young individuals vulnerable to the effects of sleep deprivation. Sleep 2009;32:999-1010.

[5] Habeck C, Rakitin BC, Moeller J, Scarmeas N, Zarahn E, Brown T. An event-related fMRI study of the neurobehavioral impact of sleep deprivation on performance of a delayed-match-to-sample task. Cogn Brain Res 2004;18:306-21.

[6] Luber B, Stanford AD, Bulow P, Nguyen T, Rakitin BC, Habeck C, et al. Remediation of sleep-deprivation induced working memory impairment with fMRI-guided transcranial magnetic stimulation. Cereb Cortex 2008; 18:2077-85.

[7] Tucker AM, Whitney P, Belenky G, Hinson JM, Van Dongen HPA. Effects of sleep deprivation on dissociated components of executive functioning. Sleep 2010;33:47-57.

[8] Chee MWL, Choo WC. Functional imaging of working memory after $24 \mathrm{~h}$ of total sleep deprivation. J Neurosci 2004;24:4560-7.

[9] Durmer JS, Dinges DF. Neurocognitive consequences of sleep deprivation. Semin Neurol 2005;25:117-29.

[10] Zarahn E, Rakitin B, Abela D, Flynn J, Stern Y. Age-related changes in brain activation during a delayed item recognition task. Neurobiol Aging 2007;28:784-98.

[11] Tucker AM, Stern Y, Basner RC, Rakitin BC. The prefrontal model revisited: double dissociations between young sleep deprived and elderly subjects on cognitive components of performance. Sleep. In Review.

[12] Steffener J, Brickman AM, Rakitin BC, Gazes Y, Stern Y. The impact of agerelated changes on working memory functional activity. Brain Imag Behav 2009;3:142-53.
[13] Habeck C, Rakitin BC, Moeller J, Scarmeas N, Zarahn E, Brown $\mathrm{T}$, et al. An event-related fMRI study of the neural networks underlying the encoding, maintenance, and retrieval phase in a delayed-match-to-sample task. Cogn Brain Res 2005;23: 207-20.

[14] Van Dongen HPA, Dinges DF. Circadian rhythms in fatigue, alertness and performance. In: Kryger MH, Roth T, Dement WC, editors. Principles and Practice of Sleep Medicine. 4th ed. Philadelphia: W. B. Saunders; 2005. p. 435-43.

[15] McGinty B, Habeck C, Hilton HJ, Rakitin B, Scarmeas N, Zarahn E. Identification and differential vulnerability of a neural network in sleep deprivation. Cereb Cortex 2004; 14:496-502.

[16] Kwong KK, Belliveau JW, Chesler DA, Goldberg IE, Weisskoff RM, Poncelet BP. Dynamic magnetic resonance imaging of human brain activity during primary sensory stimulation. Proc Natl Acad Sci U S A 1992;89:5675-9.

[17] Ogawa S, Menon RS, Tank DW, Kim SG, Merkle H, Ellerman JM. Functional brain mapping by blood oxygenation level-dependent contrast magnetic resonance imaging. A comparison of signal characteristics with a biophysical model. Biophys J 1993;64:803-12.

[18] Macwhinney B, Cohen J, Provost J. The PsyScope experiment-building system. Spat Vis 1997;11:99-101.

[19] Worsley KJ, Poline JB, Friston KJ, Evans AC. Characterizing the response of PET and fMRI data using multivariate linear models. Neuroimage 1997;6:30519.

[20] Strange BA, Portas CM, Dolan RJ, Holmes AP, Friston KJ. Random effects analysis for event-related fMRI. Neuroimage 1999;9:S36.

[21] Tzourio-Mazoyer N, Landeau B, Papathanassiou D, Crivelloa F, Etarda O, Delcroixa $\mathrm{N}$, et al. Automated anatomical labeling of activations in SPM using a macroscopic anatomical parcellation of the MNI MRI single-subject brain. Neuroimage 2002;15:273-89.

[22] Lim J, Dinges DF. Sleep deprivation and vigilant attention. Ann N Y Acad Sci 2008;1129:305-22.

[23] Chee MWL, Tan JC, Zheng H, Parimal S, Weissman DH, Zagorodnov V, et al. Lapsing during sleep deprivation is associated with distributed changes in brain activation. J Neurosci 2008;28:5519-28.

[24] Chee MWL, Tan JC. Lapsing when sleep deprived: neural activation characteristics of resistant and vulnerable individuals. Neuroimage 2010;51:83543.

[25] Drummond SPA, Bischoff-Grethe A, Dinges DF, Ayalon L, Mednick SC, Meloy MJ. The neural basis of the psychomotor vigilance task. Sleep 2005;28: 1059-68. 